\title{
Statistics of the Coulomb blockade peak spacings of a silicon quantum dot
}

\author{
F. Simmel ${ }^{1}$, David Abusch-Magder ${ }^{1,2, *}$, D. A. Wharam ${ }^{3}$, \\ M. A. Kastner ${ }^{2}$, and J. P. Kotthaus ${ }^{1}$ \\ (1) Center for NanoScience and Sektion Physik, LMU München, \\ Geschwister-Scholl-Platz 1, D-80539 München, \\ (2) Department of Physics, Massachusetts Institute of Technology, Cambridge, MA 02139, \\ (3) Institut für Angewandte Physik, Universität Tübingen, \\ Auf der Morgenstelle 10, D-72076 Tübingen
}

(July 31, 2018)

We present an experimental study of the fluctuations of Coulomb blockade peak positions of a quantum dot. The dot is defined by patterning the two-dimensional electron gas of a silicon MOSFET structure using stacked gates. This permits variation of the number of electrons on the quantum dot without significant shape distortion. The ratio of charging energy to single particle energy is considerably larger than in comparable GaAs/AlGaAs quantum dots. The statistical distribution of the conductance peak spacings in the Coulomb blockade regime was found to be unimodal and does not follow the Wigner surmise. The fluctuations of the spacings are much larger than the typical single particle level spacing and thus clearly contradict the expectation of random matrix theory.

PACS numbers: 73.23.Hk,05.45.+b,73.20.Dx

The spectral properties of many quantum mechanical systems whose classical behavior is known to be chaotic are remarkably well described by the theory of random matrices (RMT) [1]. This has been experimentally confirmed, for example, in measurements of slow neutron resonances of nuclei [2] and in microwave reflection spectra of billiard shaped cavities [3]. Electron transport experiments performed on semiconductor quantum dots in the Coulomb blockade (CB) regime [4 provide a further possibility to check RMT predictions. The classical motion of electrons in these structures can be assumed to be chaotic due to an irregular potential landscape produced by impurities, an asymmetric confinement potential [5], and/or electron-electron interactions [6]. The transport properties of quantum dots are inherently related to their energy spectra and electronic wavefunctions and thus the connection with RMT is readily made [5, 7].

Indeed, experiments on the distribution of conductance peak heights of quantum dots in the Coulomb blockade regime have shown good agreement with the predictions of RMT [8.9. On the other hand, the distribution of the CB peak spacings have been found to deviate from the expectations of RMT 10 12. The results suggest that the peak spacings are not distributed according to the famous Wigner surmise. Furthermore, there is no indication of spin degeneracy which would result in a bimodal peak spacing distribution [12]. In Refs. 110,11 the fluctuations of the peak spacings are considerably larger than expected from RMT, whereas the experiments presented in Ref. 12 yield smaller peak spacing fluctuations, which, however, are still larger than those predicted by RMT.

The deviations from the RMT predictions have been frequently interpreted as fluctuations in the charging energy. As the charging energy reflects the Coulomb interactions both between the electrons on the dot as well as between the dot and its environment, the dependence of the fluctuations on the interaction strength is of fundamental interest. Numerical studies suggest that the fluctuations are proportional to the charging energy rather than to the single particle level spacing 10,13,14. This is also found theoretically for the classical limit of a Coulomb glass island [15. In RPA calculations the fluctuations have been related to fluctuations of the eigenfunctions of the dot [16]. Another approach based on density functional theory emphasizes the role of the Coulomb matrix elements of scarred wavefunctions [17. Recently, also a non-interacting explanation for the Gaussian shape of the peak spacing distribution has been given in terms of level dynamics due to shape deformation of the quantum dot [18].

Here we present an experimental study of the statistics of Coulomb blockade peak positions of a quantum dot. The dot is defined by patterning the twodimensional electron gas of a silicon metal oxide semiconductor field effect transistor (MOSFET) structure using stacked gates. These experiments differ significantly in two major ways from prior experiments on quantum dots defined in GaAs/AlGaAs heterostructures: first and foremost, due to the different electron density and material properties of silicon, the ratio of the charging energy, $E_{C}$, to the single particle energy level spacing, $\Delta \epsilon$, is considerably larger; likewise the dimensionless parameter $r_{s}$, which characterizes the strength of the Coulomb interactions, is larger than in previous studies. Secondly, the number of electrons is varied by the application of a voltage to a top gate instead of by squeezing the quantum dot with a plunger gate. We find that the distribution of the peak spacings is unimodal and roughly Gaussian. The magnitude of the fluctuations is 15 times larger than that predicted by RMT.

Conduction through a small electron island coupled to leads via tunnel barriers is normally suppressed if $k_{B} T \ll$ $E_{C}$, where $E_{C}$ is the charging energy of the island. This 
effect is known as the Coulomb blockade $\llbracket$. The blockade is lifted when the condition $\mu_{d}<\mu_{d o t}<\mu_{s}$ is satisfied, where $\mu_{s}, \mu_{d}$, and $\mu_{d o t}$ are the chemical potentials of the source, drain and dot, respectively. In the linear response regime, where these experiments have been performed, $\left|\mu_{d}-\mu_{s}\right| \ll \Delta \epsilon, k_{B} T$. The chemical potential of the dot is defined as $\mu_{d o t}(N+1)=E(N+1)-E(N)$ where $E(N)$ is the total energy of the dot occupied by $N$ electrons. In the case where the blockade is lifted an electron can tunnel from the source onto the dot, changing the dot's occupation from $N$ to $N+1$, and sequentially tunnel off the dot to the drain leaving the dot in its original state. The resulting fluctuation of the electron number on the dot leads to a finite conductance. Experimentally this can be achieved by appropriately tuning $\mu_{\text {dot }}$ with an external gate. Sweeping the gate voltage, $V_{g}$, results in the well known conductance oscillations indicating successive filling of the dot with single electrons. The difference of $\mu_{d o t}$ between two adjacent conductance maxima is thus given by $\Delta \mu_{N}=E(N+1)-2 E(N)+E(N-1)$ which can be viewed as the discrete second derivative of the quantum dot energy with respect to particle number, i.e. the inverse compressibility $\partial \mu / \partial N$ 13].

In the constant interaction (CI) model [4] the energy of the dot is approximated as $E(N)=(N e)^{2} / 2 C_{\Sigma}+$ $\sum_{i=1}^{N} \epsilon_{i}$, where the electrostatic interactions are treated using a simple capacitive charging model with a total dot capacitance $C_{\Sigma}$, and the quantum mechanical terms are taken into account as single particle energies $\epsilon_{i}$. In this model the difference of the chemical potentials for successive occupation numbers, the so called addition energy, is $\Delta \mu_{N}=E_{C}+\Delta \epsilon_{N}$ with the charging energy $E_{C}=e^{2} / C_{\Sigma}$, and the level spacing $\Delta \epsilon_{N}=\epsilon_{N+1}-\epsilon_{N}$. This is mapped to gate voltages via $e \frac{C_{g}}{C_{\Sigma}} \Delta V_{g}=E_{C}+\Delta \epsilon_{N}$ where $C_{g}$ is the capacitance of the dot to the gate and $\Delta V_{g}$ the difference between the gate voltages at which adjacent conductance maxima occur.

This final expression motivated the original investigations of the peak spacings in the light of random matrix theory. RMT shows that the normalized spacings $S$ $(\langle S\rangle=1)$ between adjacent eigenvalues of a generic timereversal invariant Hamiltonian are distributed according to the Wigner surmise

$$
P_{W}(S)=\frac{\pi}{2} S e^{-\frac{\pi}{4} S^{2}} .
$$

The fluctuations of these spacings are $\left(\left\langle S^{2}\right\rangle-\langle S\rangle^{2}\right)^{1 / 2} \approx$ $0.52\langle S\rangle$. However, experiments have shown that the combined CI-RMT model is not capable of describing the observed peak spacing distribution correctly 10 12.

The Coulomb blockade measurements on which the following analysis is based have been performed on a quantum dot defined in a silicon MOSFET structure. We have utilized a stacked gate structure to pattern the electron gas as shown in Fig. 1. First, a gate oxide is grown on a p-type silicon substrate (lower oxide), and then a lower metal gate is deposited and patterned using electron beam lithography and lift-off techniques. Above the lower gate a second layer of silicon dioxide is deposited (upper oxide), and finally an upper gate is formed; the upper oxide layer serves to insulate the lower gate from the upper gate. Application of positive voltages to the upper gate leads to the formation of a two-dimensional electron gas (2DEG) at the $\mathrm{Si} / \mathrm{SiO}_{2}$ interface; $n^{+}$implanted regions serve as Ohmic contacts to the 2DEG. Further details about this device may be found elsewhere 19,20 . The lower gates locally screen the electric field created by the upper gate, and a quantum dot is formed by applying appropriate negative voltages to the lower gates. The size of the dot is estimated from the capacitance to be $A \approx 200 \mathrm{~nm} \times 200 \mathrm{~nm}$, which agrees well with the lithographic dimensions of $250 \mathrm{~nm} \times 270 \mathrm{~nm}$ when electrostatic depletion at the edge is considered. The electron density can be varied by changing the upper gate voltage, whereas the lower gate voltage controls the tunnel barriers and the electrostatic confinement potential of the quantum dot. This technique allows the definition of very small structures which therefore have low capacitances and high charging energies. For the quantum dot discussed here these values are $C_{\Sigma} \approx 85 \mathrm{aF}$ and $E_{C} \approx 1.9 \mathrm{meV}$ as obtained from temperature dependence measurements of the conductance resonances [20].

In contrast to previous experiments on quantum dots in GaAs/AlGaAs heterostructures the electron density is considerably higher, $n_{s} \approx 2.5 \cdot 10^{16} \mathrm{~m}^{-2}$. The mobility of the two-dimensional electron gas is $\mu=0.56 \mathrm{~m}^{2} / \mathrm{Vs}$, and the mean free path $l \approx 100 \mathrm{~nm}$ is comparable to the system size. The single particle energy level spacing can be obtained from the estimated dot area, $A$, via Weyl's formula 21] as $\Delta \epsilon=\frac{2 \pi \hbar^{2}}{g m^{*} A}=15 \mu \mathrm{eV}$, where $g$ is the degeneracy of electronic states in the two-dimensional electron gas, and $m^{*}$ is the effective mass of the electrons. In a 2DEG in a silicon MOS system $m^{*}=0.2 m_{e}$, and at $B=0$ both the spin and valley degeneracies must be considered and therefore $g=4$. While these quantum dots are smaller than many of the GaAs/AlGaAs quantum dots studied [10 12], $\Delta \epsilon$ is of the same order due to the larger effective mass, and to the valley degeneracy.

The strength of the electronic interactions characterized by the dimensionless parameter $r_{s}=$ $g /\left(2 \sqrt{\pi n_{s}} a_{B}^{*}\right)=2.1$ considerably exceeds the values obtained in recent experiments (where $r_{s} \approx 1$ ) 10 12]; here $a_{B}^{*}$ is the effective Bohr radius. Similarly, the ratio of charging energy to single particle energy level spacing $E_{C} / \Delta \epsilon \approx 125$, another measure of the relative importance of electron-electron interactions, is larger than in the experiments performed on GaAs/AlGaAs quantum dots.

The measurements were performed in a ${ }^{3} \mathrm{He}$ refrigerator at a temperature of $T=320 \mathrm{mK}$ using standard lock-in techniques at low frequencies and bias. The conductance oscillations were measured as a function of the upper gate voltage. Consequently, the electron density was varied without drastically changing other system parameters such as charging energy, single particle energy, 
and dot shape. This also contrasts with former experiments on the statistics of conductance oscillations where the shape of the quantum dot was distorted by plunger gates 9,12$]$.

The following analysis is based on a series of more than 100 conductance peaks occurring in the upper gate voltage range from $12.1 \mathrm{~V}$ to $13.5 \mathrm{~V}$ (see Fig. 2 set). In this range the electron density changes from $2.4 \times 10^{16} \mathrm{~m}^{-2}$ to $2.6 \times 10^{16} \mathrm{~m}^{-2}$. The quantum point contacts connecting the quantum dot to the leads are tuned into the tunneling regime by applying voltages of $-4.5 \mathrm{~V}$ and $-8.0 \mathrm{~V}$ to the left and right pair of lower gates, respectively. The position of each peak is obtained by fitting the peak by a thermally broadened line shape $\propto \cosh ^{-2}\left(e \alpha\left(V-V_{0}\right) / 2 k_{B} T\right)$ [4] where $\alpha$ and $V_{0}$ serve as the fit parameters. The gate voltage spacings $\Delta V_{g}$ are calculated from the peak positions.

The mean value $\left\langle\Delta V_{g}\right\rangle$ of the spacings as a function of $V_{g}$ is obtained from a linear fit as $\Delta V_{g}\left(V_{g}\right) \approx$ $\left[12.2-\frac{3.5 \cdot 10^{-2}}{\mathrm{~V}} \times\left(V_{g}-12.5 \mathrm{~V}\right)\right] \mathrm{mV}$. The smallness of the slope of this fit shows that the influence of the upper gate on the capacitance and therefore on the size of the dot is rather weak. Accordingly, the shape deformation which has been postulated to explain the distribution of $\Delta V_{g}$ 18] plays no significant role in this experiment. The normalized peak spacings

$$
\delta=\frac{\Delta V_{g}-\left\langle\Delta V_{g}\right\rangle}{\left\langle\Delta V_{g}\right\rangle}
$$

are displayed in Fig. 2. The fluctuations of $\delta$ are computed to be $\left\langle\delta^{2}\right\rangle^{1 / 2} \approx 0.06$. The fluctuations in the addition energy are, therefore, roughly $115 \mu \mathrm{eV}$, which is 7.5 times the mean level spacing $\Delta \epsilon$, and thus 15 times larger than the fluctuations expected from RMT. This supports the view that the fluctuations of the addition energy scale with the Coulomb energy rather than with the kinetic energy. However, the proportionality factor 0.06 is smaller than that suggested by numerical calculations $(0.1-0.2)$ [14. It should be noted that in these experiments $\Delta \epsilon \approx k_{B} T$. We expect that the effect of thermal broadening would be to reduce the fluctuations in peak spacing. A simple model [12] predicts that the fluctuations expected within RMT would be reduced by a factor of $2-3$. If we incorporate this correction into RMT then the fluctuations we find in our experiment are 30 - 45 times larger than those predicted by RMT.

The distribution of the peak spacings normalized to an area of unity is shown in Fig. 3. The distribution is unimodal and roughly has the shape of a Gaussian. In the inset of Fig. 3 the experimental distribution is depicted together with the Wigner surmise (Eq. 1); for comparison to $\delta$ we have rescaled the predictions of RMT taking into account the experimental values of $E_{C}$ and $\Delta \epsilon$. As in previous experiments there is no evidence of a bimodal addition spectrum as is predicted by the CI-RMT model. This is in agreement with the theoretical prediction that the influence of spin degeneracy on the addition spectrum is washed out for stronger electron-electron interactions $\left(r_{s}>1\right)$ 14, 16.

In conclusion, we have investigated the Coulomb blockade peak spacing distribution of a quantum dot fabricated in the 2DEG of a silicon MOSFET structure. In accordance with experiments on GaAs/AlGaAs quantum dots the distribution differs from the Wigner surmise and is roughly Gaussian. The fluctuations are approximately $0.06 \times E_{C}$. Due to the large ratio of charging energy $E_{C}$ to single particle energy $\Delta \epsilon$ this strongly suggests that the fluctuations scale with $E_{C}$ and not with $\Delta \epsilon$. This clearly contradicts the predictions of RMT and indicates that the fluctuations are dominated by electron-electron interactions in this system.

* new address: Bell Laboratories, Lucent Technologies, 600 Mountain Ave., Murray Hill NJ 07974

\section{ACKNOWLEDGMENTS}

The authors would like to thank the staff of the Microelectronics Lab at Lincoln Laboratory for their help with device fabrication, and U. Sivan, M. Stopa, H. Baranger for fruitful discussions. This work was supported by the Alexander-von-Humboldt Foundation, the DFG Sonderforschungsbereich 348, and by the Army Research Office under contract DAAH04-94-0199 and contract DAAH0495-0038. Work at Lincoln Laboratory was sponsored by the U.S. Air Force. DA-M gratefully acknowledges support from the Alexander-von-Humboldt Foundation, and the Bell Labs Foundation.

[1] M. L. Mehta, Random Matrices, 2nd ed. (Academic Press, London, 1991).

[2] R. U. Haq, A. Pandey, and O. Bohigas, Phys. Rev. Lett. 48, 1086 (1982).

[3] H. D. Gräf, H. L. Harney, H. Lengeler, C. H. Lewenkopf, C. Rangacharyulu, A. Richter, P. Schardt, and H. A. Weidenmüller, Phys. Rev. Lett. 69, 1296 (1992).

[4] There are several recommendable review articles about the Coulomb blockade phenomenon in semiconductor quantum dots, e.g. H. van Houten, C. W. J. Beenakker, and A. A. M. Staring in Single Charge Tunneling, edited by H. Grabert, J. M. Martinis, and M. H. Devoret (Plenum, New York, 1991); U. Meirav and E. B. Foxman, Semicond. Sci. Technol. 10, 255 (1995); L. P. Kouwenhoven, C. M. Marcus, P. L. McEuen, S. Tarucha, R. M. Westervelt, and N. S. Wingreen, in Mesoscopic Electron Transport, edited by L. L. Sohn, L. P. Kouwenhoven, and G. Schön, NATO ASI Series E (Kluwer, Dordrecht, 1997).

[5] R. A. J. Jalabert, A. D. Stone, and Y. Alhassid, Phys. Rev. Lett. 68, 3468 (1992). 
[6] L. Meza-Montes and S. E. Ulloa, Phys. Rev. E 55, R6319 (1997).

[7] C. W. J. Beenakker, Rev. Mod. Phys. 69, 731 (1997).

[8] A. M. Chang, H. U. Baranger, L. N. Pfeiffer, K. W. West, and T. Y. Chang, Phys. Rev. Lett. 76, 1695 (1996).

[9] J. A. Folk, S. R. Patel, S. F. Godijn, A. G. Huibers, S. M. Cronenwett, C. M. Marcus, K. Chapman, and A. C. Gossard, Phys. Rev. Lett. 76, 1699 (1996).

[10] U. Sivan, R. Berkovits, Y. Aloni, O. Prus, A. Auerbach, and G. Ben-Yoseph, Phys. Rev. Lett. 77, 1123 (1996).

[11] F. Simmel, T. Heinzel, and D. A. Wharam, Europhys. Lett. 38, 123 (1997).

[12] S. R. Patel, S. M. Cronenwett, D. R. Stewart, A. G. Huibers, C. M. Marcus, C. I. Duruöz, J. S. Harris Jr., K. Champman, and A. C. Gossard, Phys. Rev. Lett. 80, 4522 (1998).

[13] R. Berkovits and B. L. Altshuler, Phys. Rev. B 55, 5297 (1997).

[14] R. Berkovits, Phys. Rev. Lett. 81, 2128 (1998).

[15] A. A. Koulakov, F. G. Pikus, and B. I. Shklovskii, Phys. Rev. B 55, 9223 (1997).

[16] Y. M. Blanter, A. D. Mirlin, and B. A. Muzykantskii, Phys. Rev. Lett. 78, 2449 (1997).

[17] M. Stopa, Physica B 249-251, 228 (1998).

[18] R. O. Vallejos, C. H. Lewenkopf, and E. R. Mucciolo, Phys. Rev. Lett. 80, 677 (1998).

[19] David Abusch-Magder, M.A. Kastner, C.L. Dennis, W.F. DiNatale, T.M. Lyszczarz, D.C. Shaver, and P.M. Mankiewich, in Quantum Transport in Semiconductor Submicron Structures, edited by B. Kramer, NATO ASI Series E 326 (Kluwer, Dordrecht, 1996), p. 251.

[20] David Abusch-Magder, Ph.D. thesis, Massachusetts Institute of Technology, 1997.

[21] H. P. Baltes and E. R. Hilf, Spectra of Finite Systems: A Review of Weyl's Problem, Bibliographisches Institut, Zürich (1976).

FIG. 1. Schematic representation of the device design. A cross section of the device is shown in (a). Two oxide and two gate layers are formed on top of p-type silicon substrate. The lower oxide layer has a thickness of roughly $20 \mathrm{~nm}$, while the upper oxide is approximately $80 \mathrm{~nm}$ thick. The voltage on the upper gate is used to vary the electron density in the 2DEG induced at the interface of the lower oxide and the silicon. A top view of the device is shown in (b). The pattern in the lower gates defines a quantum dot in the induced electrons; note that the upper gate covers all of the area show in (b) and overlaps the source and drain. The lithographic dimensions of the quantum dot are $250 \mathrm{~nm} \times 270 \mathrm{~nm}$.

FIG. 2. The normalized peak spacings $\delta$ obtained from an upper gate voltage sweep. The fluctuations around the mean value 0 are much larger than expected from the CI-RMT model. The inset shows Coulomb blockade conductance oscillations as a function of the upper gate voltage.
FIG. 3. Histogram showing the distribution of the normalized peak spacings from Fig. 2. The area of the histogram is normalized to unity. A Gaussian fit with standard deviation of $\sigma=0.06$ is also displayed. The inset shows the same histogram alongside the Wigner surmise, the distribution predicted by RMT, for $\Delta \epsilon=15 \mu \mathrm{eV}$. The experimental distribution is much broader than expected from RMT. 
(a)

upper gate

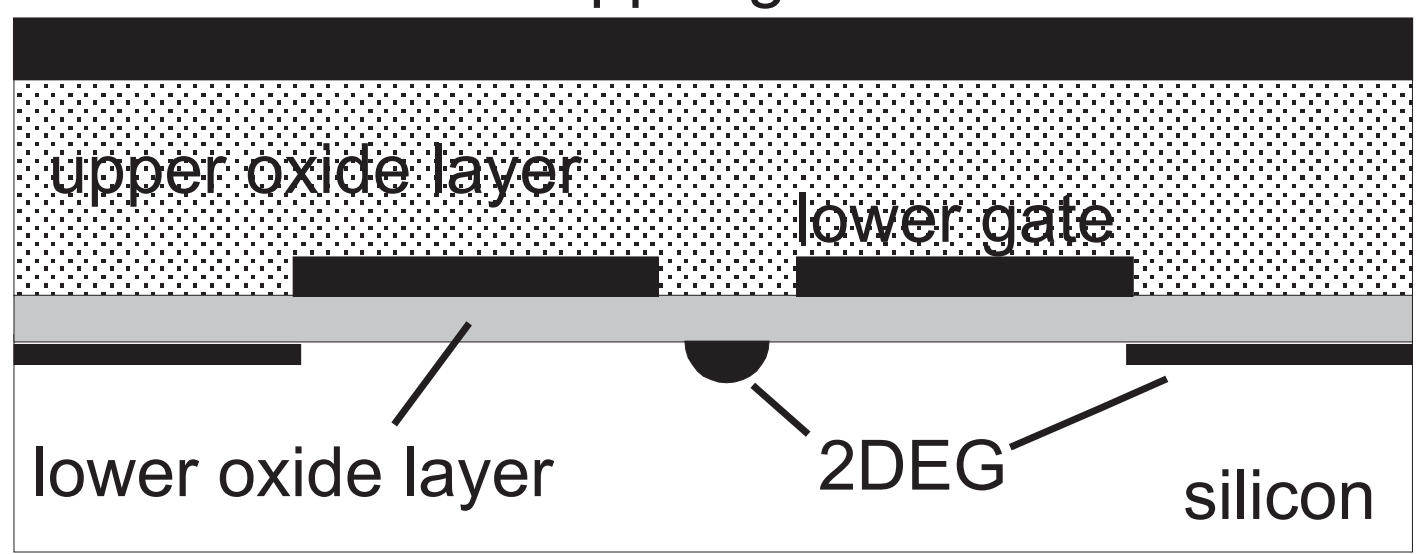

(b)
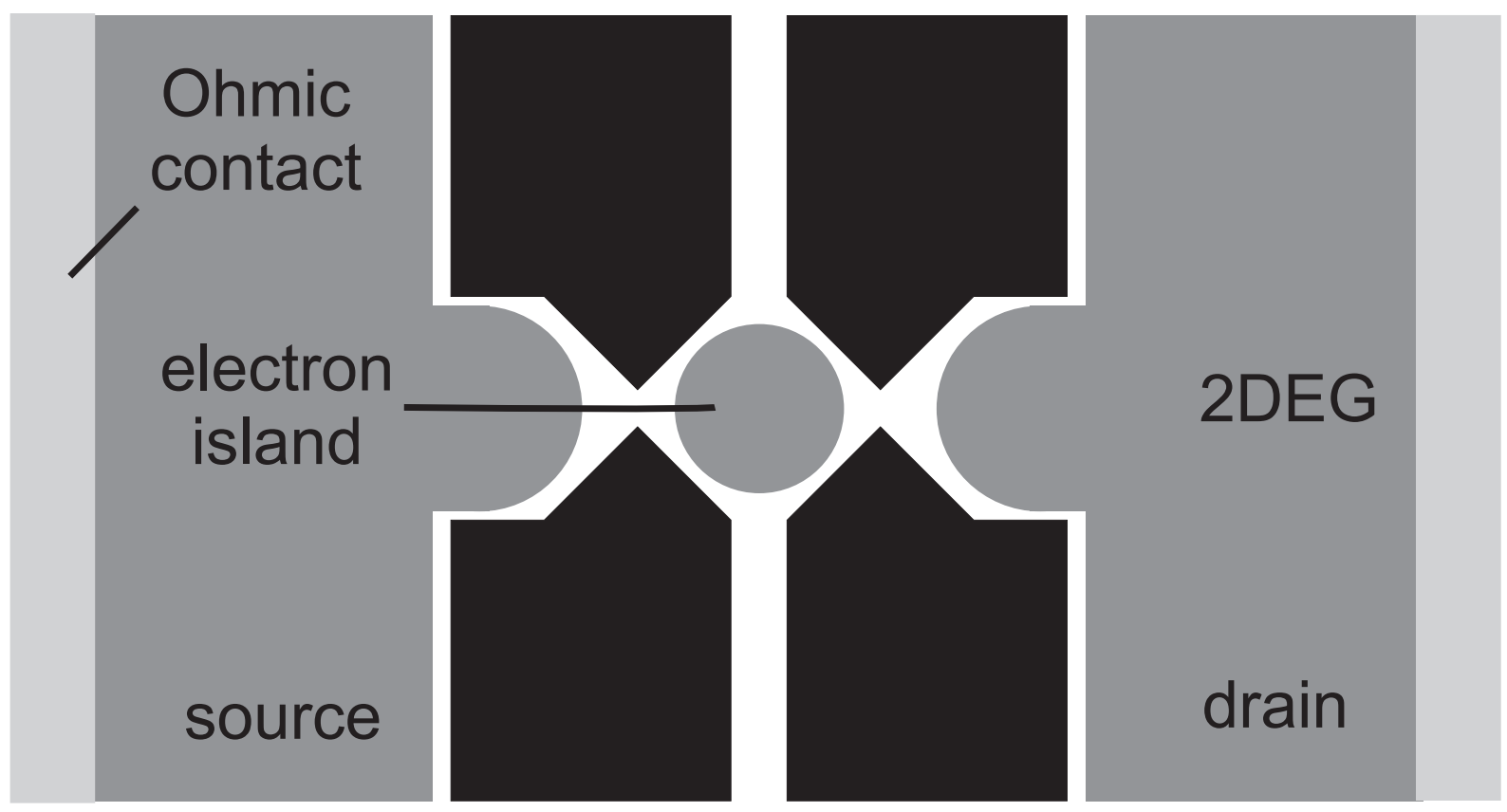

lower gate 


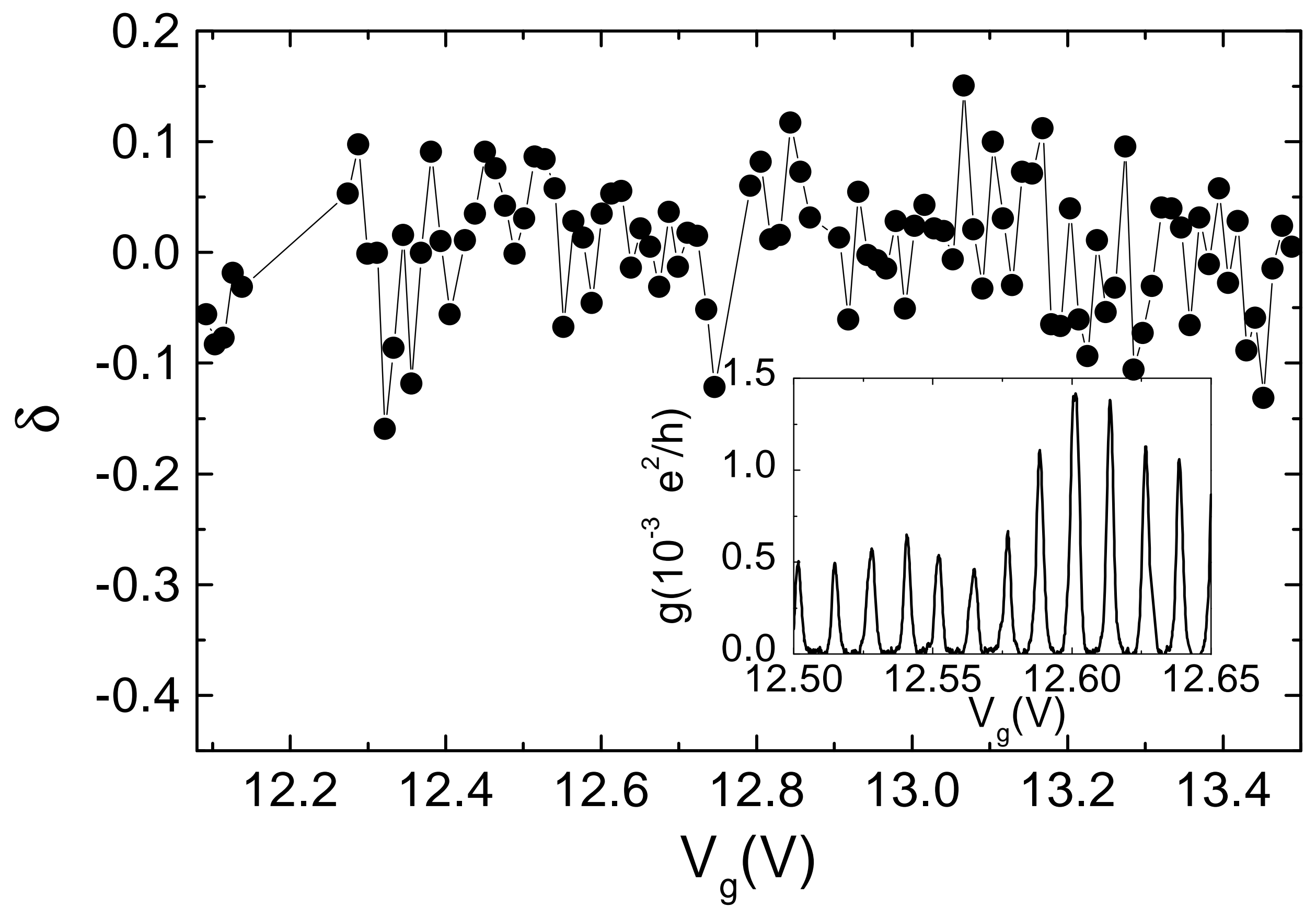




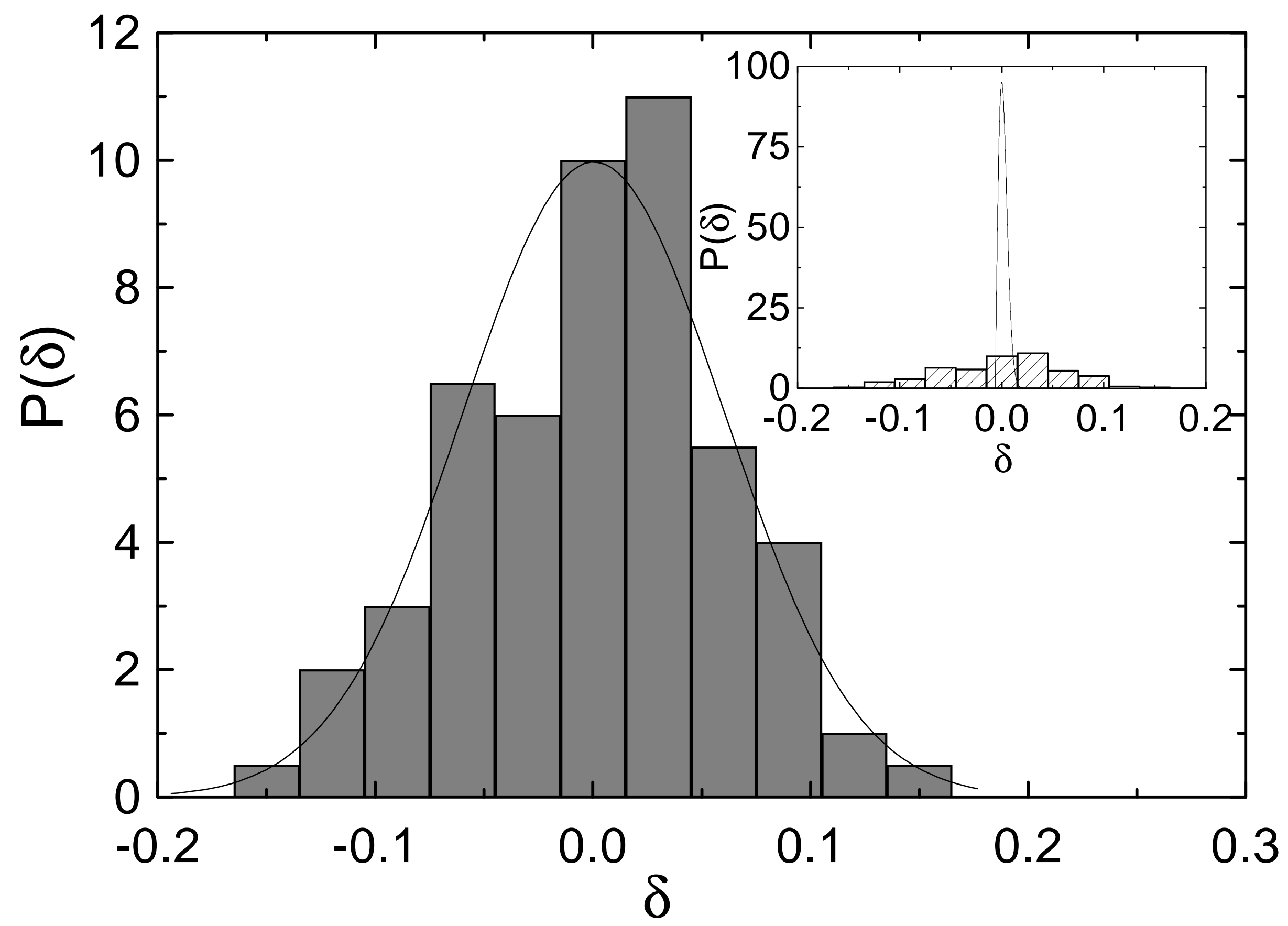

